\title{
Surgical Management of the Infected Femoral Pseudoaneurysms In Intravenous Drug Addicts
}

\author{
Ashraf Mohammed Ewida, Yasser Hussin Hassan Metwally, Mohammed Abdessalam \\ Abdallah Hemdan
}

\author{
Department of Vascular Surgery, Faculty of Medicine, Al-Azhar University, Cairo, Egypt. \\ Corresponding author: Mohammed Abdessalam Abdallah Hemdan,email: abdessalam101n@gmail.com
}

\begin{abstract}
Background: Vascular system complications are an ongoing hazard of intravenous drug abuse.

Patients and Method: This is a prospective study. The study was conducted on twenty patients who were presented at AL Hussein University Hospital in Cairo with infected femoral artery pseudoaneurysm over a period of eight months from Nov 2017 to July 2018. The inpatient medical charts were reviewed. Data were obtained on their demographic, clinical, management and outcome parameters.

Result: Among the twenty intravenous drug users presenting with infected femoral artery pseudoaneurysms, (16) of the patients $(80 \%)$ were seropositive for HCV. All were operated, with (5\%) mortality. Primary ligation and debridement was the technique used almost exclusively (75\%). One patient was managed by primary reconstruction by direct in-situ repair. Four patients were managed by primary revascularization through extaanatomical bypass. Mean follow up period was three months.

Conclusion: Ligation and debridement appears to be a simple, safe and effective procedure in infected pseudoaneurysms in IV drug users.
\end{abstract}

Keywords: IV drug users, infected pseudoaneurysms, revascularization, ligation.

\section{INTRODUCTION}

IV drug abuse is an international sociomedical problem. The number of drug users was increasing in recent years. It was estimated that about 14 million people worldwide injected illicit drugs in $2011^{(\mathbf{1})}$. Femoral triangle is a common site for illegal substances injection with accidental puncturing of the common femoral artery. Vascular system complications are an ongoing hazard of intravenous drug abuse. Repeated failed attempts to inject preparations as heroin and cocaine into a major lower limb deep vein are likely to cause arterial or venous injury. Pseudoaneurysm is a pulsating, encapsulated hematoma in communication with the lumen of a ruptured artery ${ }^{(\mathbf{1}, \mathbf{2})}$.

These patients are usually presented with painful groin swelling with bleeding puncture site. Pulsating and tender swelling is commonly seen in examination. If left untreated, the infected pseudoaneurysm may be accompanied by systemic sepsis, life-threatening hemorrhage, loss of limb or even death. Various other vascular complications, such as deep venous thrombosis (DVT) and arterial embolization can occur ${ }^{(2)}$. Different diagnostic modalities are used for primary diagnosis and intraoperative assessment. In addition, postoperative and long term follow-up such as imaging options as duplex US ${ }^{(3,4)}$, CT, CT angiography, MRA in selected cases and bedside tests as Doppler signals detection, digital PO2 by pulse oximeter and ABI evaluation ${ }^{(2,5,6,7)}$.. The treatment aims are firstly to control actual or potential hemorrhage by oversewing or ligating rupture arteries; secondly, to excise and drain the local septic tissue. Controversy exists as to which is the appropriate way to manage infected femoral pseudoaneurysm ligation or revascularization ${ }^{(2,8)}$. Trans-obturator bypass is a valuable option of revascularization so, vascular surgeons may need to become more familiar with this procedure and consider performing it in appropriate cases ${ }^{(9)}$.

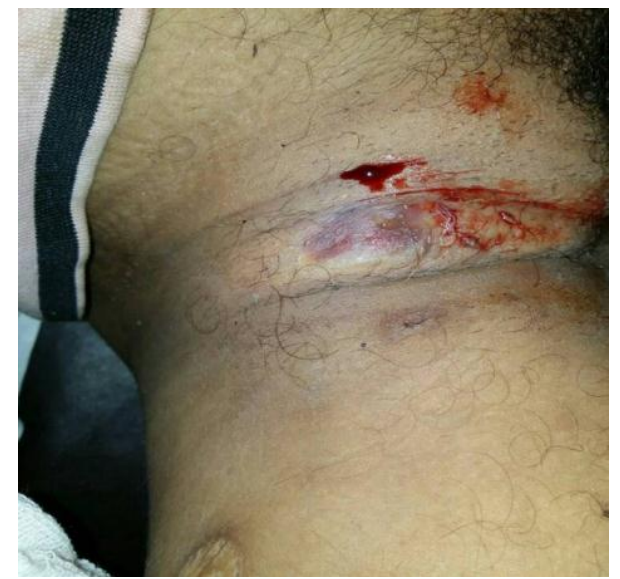

Fig. (1): Right infected femoral artery pseudoaneurysm with ongoing bleeding.

\section{PATIENTS AND METHODS}

This study was conducted on twenty patients who were presented at AL Hussein University Hospital in Cairo with infected femoral artery pseudoaneurysm over a period of eight months from Nov 2017 to July 2018. Upon clinical suspicion, all underwent duplex US of the concerned limb except a patient who was massively bleeding at presentation and had to be 
rushed to operating room. Emergent surgery was planned in each of them as soon as diagnosis was made. All the patients received IV broad-spectrum antibiotics at presentation to be directed later on according to culture and sensitivity. Full laboratory investigations and virology markers were done. Different diagnostic modalities were used for primary diagnosis and pre-operative assessment as duplex US, CT, CT angiography(CTA) and MRA in selected cases. In addition, bedside tests as Doppler signals detection, digital $\mathrm{PO} 2$ by pulse oximeter and ankle-brachial pressure index (APBI) evaluation were done. Intraoperative assessment by intraoperative selective angiography if available and bedside tests. Postoperative and long term follow-up by clinical assessment, duplex US, CTA and bedside tests. Surgical strategies included: (a) Ligation of the affected arterial segment and debridement of all necrotic and infected tissue without revascularization that is preserved for patients who underwent primary ligation and experienced postoperative manifestations of acute ischaemia (b) Ligation and debridement with primary arterial revascularization.

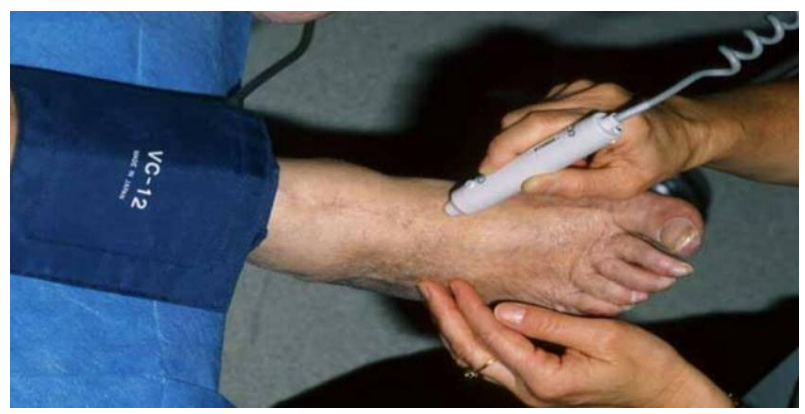

Fig. (2): ABPI assessment.

\section{RESULTS}

Twenty patients with history of chronic intravenous drug abuse presented to the emergency room with infected femoral artery pseudoaneurysm. 16 patients $(80 \%)$ were seropositive for $\mathrm{HCV}$ and the remaining were seronegative. 14 patients $(70 \%)$ were diagnosed by duplex US and 2 patients $(10 \%)$ were diagnosed by CTA because of aneurysmal thrombosis and duplex was not conclusive. 4 patients (20\%) were presented with ruptured infected femoral artery pseudoaneurysm (IFAP), who were haemodynamically unstable and not candidates for any pre-operative vascular imaging. Staphyllococcus aureus was the most common isolated organism representing 11 patients (55\%), four of them (20\%) had methicillin-resistant Staphylococcus aureus
(MRSA), Pseudomonas auroginosa represented (20\%), Streptococcus pyogenes represented (15\%), one culture $(5 \%)$ revealed mixed infections and one culture $(5 \%)$ showed no growth.

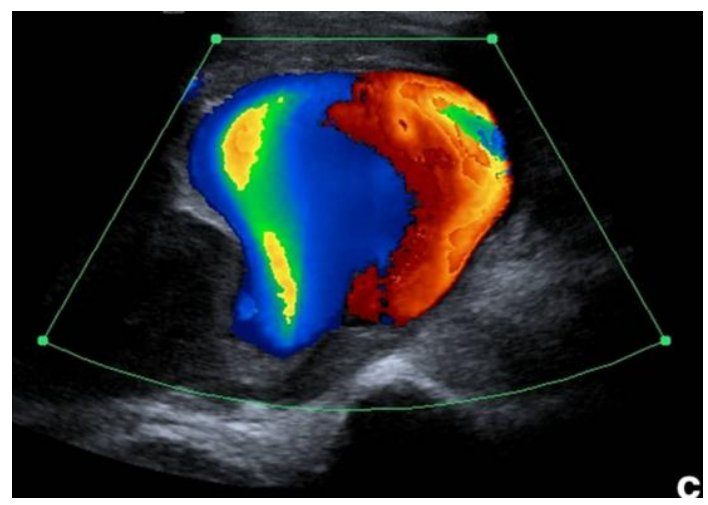

Fig. (3): Duplex US: Yin-Yang sign.

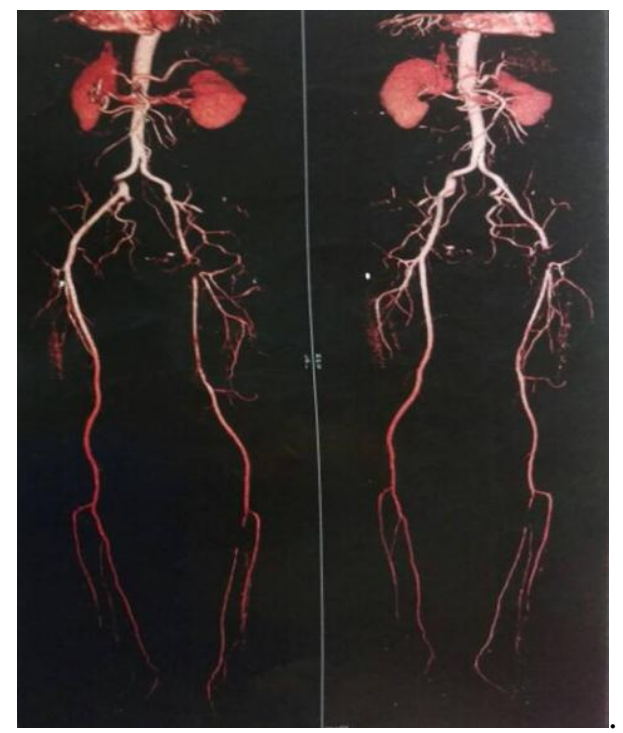

Fig. (4): CTA: left CFA injury due to injection.

Table(1): Isolated organisms by cultures.

\begin{tabular}{|l|c|c|}
\hline \multirow{2}{*}{\multicolumn{1}{c|}{ Organism }} & \multicolumn{2}{c|}{ Patients } \\
\cline { 2 - 3 } & No. & \% \\
\hline Staphylococcus aureus & 7 & $35 \%$ \\
\hline MRSA & 4 & $20 \%$ \\
\hline Pseudomonas auroginosa & 4 & $20 \%$ \\
\hline Streptococcus pyogens & 3 & $15 \%$ \\
\hline No growth & 2 & $10 \%$ \\
\hline
\end{tabular}

$(\mathrm{n}=15 ; 75 \%)$ patients were managed by primary ligation, $(\mathrm{n}=1 ; 5 \%)$ underwent in-situ reconstruction by venous patch that complicated with groin infection and sartorius muscle flap was done and $(\mathrm{n}=4 ; 20 \%)$ patients were managed by primary revascularization through extra-anatomical bypass as they were presented in acute ischaemia. All patients with $\mathrm{PO} 2$ values lower than $90 \%$ needed revascularization after ligation. Patients with $\mathrm{ABPI}$ and 
paedal Doppler waves suggesting chronic ischaemia were more resistant after ligation and all of them passed without revascularization. During the period of follow up, $(\mathrm{n}=5 ; 25 \%)$ of patients experienced post-ligation acute ischaemia and underwent revascularization through extra-anatomical bypass, $(\mathrm{n}=3 ; 15 \%)$ of patients underwent above knee amputation, $(n=1 ; 5 \%)$ died of septic shock due to infected above knee stump.

All of extra-anatomical bypasses done were through the lateral femoral route and all done with $8 \mathrm{~mm}$ PTFE synthetic graft. Patients with revascularization compared to patients with ligation had more documented complications, more re-operation rate and longer hospital stay of ratio $(2: 1),(3: 1)$ and (5:2), respectively.

Table (2): The primary performed procedures.

\begin{tabular}{|l|c|c|}
\hline \multirow{2}{*}{\multicolumn{1}{|c|}{ Primary procedure }} & \multicolumn{2}{|c|}{ Patients } \\
\cline { 2 - 3 } & No. & \% \\
\hline $\begin{array}{l}\text { Ligation without } \\
\text { revascularization }\end{array}$ & 15 & $75 \%$ \\
\hline $\begin{array}{l}\text { Ligation with primary } \\
\text { revascularization }\end{array}$ & 4 & $20 \%$ \\
\hline In-situ reconstruction & 1 & $5 \%$ \\
\hline
\end{tabular}

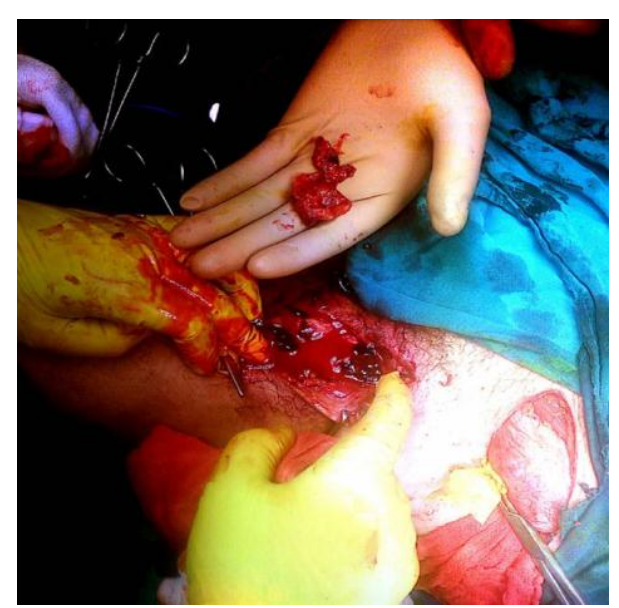

Fig. (5): Debridement of IFAP with necrotic femoral vessels.

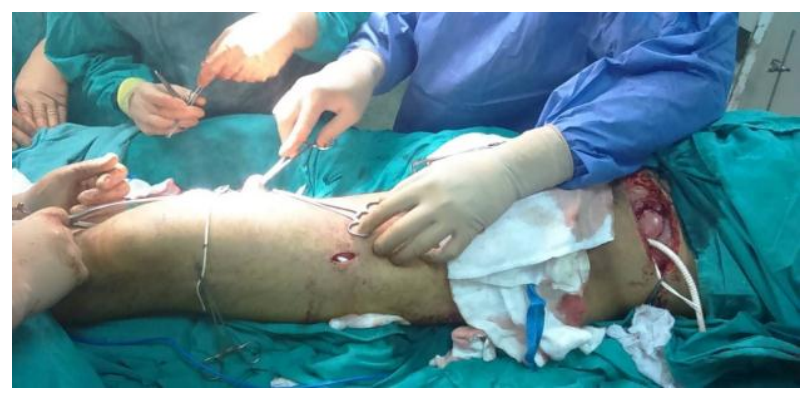

Fig. (6): Revascularization through ilio(CIA)-popliteal extraanatomical lateral femoral bypass by PTFE synthetic graft.
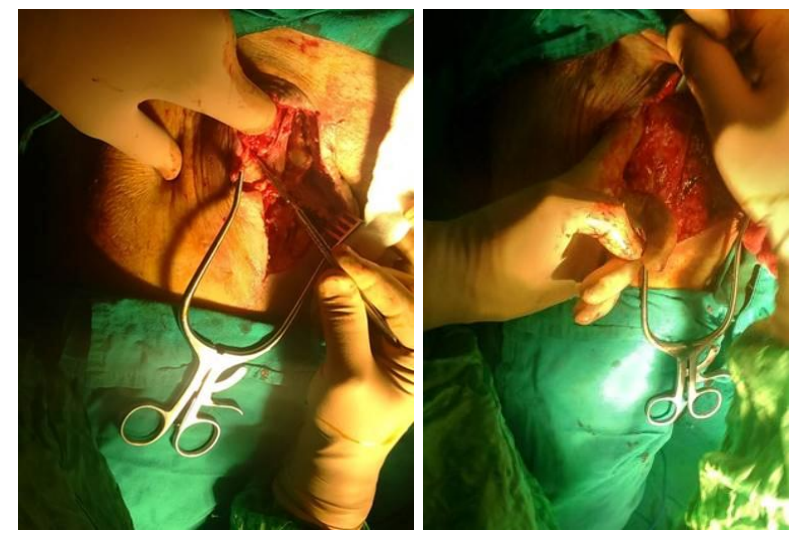

Fig. (7): Sartorius muscle flap procedure.

\section{DISCUSSION}

Femoral triangle is a common site for illegal substances injection with accidental puncturing of the common femoral artery. Qiu et al. ${ }^{(2)}$ reported that infected femoral artery pseudoaneurysms (IFAPs) accounts for approximately $0.14 \%$ of the complications associated with the injection of intravenous drugs.

Viral infection is common among these patients because of needle sharing habits. Staphyllococcus aureus is the most common organism causing femoral pseudoaneurysm infection in drug abusers. In a study conducted by Luther et al. (1) on fifty patients with IFAPs, Staphylococcus aureus was the most common isolated organism representing (48\%). Duplex US and CTA were valuable imaging modalities for diagnosis of IFAP. Stone and Hass ${ }^{(3)}$ concluded that Sensitivity and specificity of duplex US for detection of pseudoaneurysm were high at $95 \%$ and $95 \%$. Molina et al. (7) concluded that CT angiography had a sensitivity and specificity of $95.1 \%$ and $98.7 \%$ respectively, in detecting traumatic pseudoaneurysms in the proximal extremities.

Patients presented with chronic ischaemic limbs experienced less ischaemic manifestations and these limbs were more resistant after ligation. Qiu et al. ${ }^{(2)}$ concluded that patients with chronic ischaemia due to long standing and frequent intraarterial injection have well-established collaterals that make them more resistant to ligation hazards. Groin infection over in-situ arterial repair should be managed seriously and soft tissue coverage should be considered, Jayaraman et al. (10) recommended. Lateral femoral bypass technique was more frequent to be done than the trans- 
obturator type as it was easier and simpler. The author recorded four cases other than who were included in this study, were managed by transobturator method and concluded that they were less in graft infection rate, higher in primary patency and less associated to re-injection. Ligation has less complications, more safe and less time consuming procedure than revascularization.

\section{CONCLUSION}

Pseudoaneurysms in IV drug abusers present frequently in an infected state as patients are usually late to seek medical advice by several days to weeks. Patients with chronic ischaemia at presentation have well-established collaterals and are less affected after ligation than others. Ligation and debridement is a simple, safe and effective procedure with minimal morbidity. Trans-obturator extra-anatomical bypass seems to be better than lateral femoral type, although it is technically more difficult.

\section{REFERENCES}

1. Luther A, Kumar A, Negi KN (2015): Peripheral Arterial Pseudoaneurysms a 10Year Clinical Study. Indian Journal of Surgery, 77: S603-S607

2. Qiu J, Zhou W, Zhou W et al. (2016): The Treatment of Infected Femoral Artery Pseudoaneurysms Secondary to Drug Abuse: 11 years of experience at a single institution. Annals of Vascular Surgery, 36: 35-43

3. Stone PA and Hass SM (2014): Vascular Laboratory: Arterial Duplex Scanning. Cronenwett JL. and Johnston KW:Rutherford's Vascular Surgery, eighth edition, Philadelphia.

4. Montorfano MA, Pla F, Vera L et al. (2017): Point of care ultrasound and Doppler ultrasound evaluation of vascular injuries in penetrating and blunt trauma. Critical Ultrasound Journal. 9:5

5. Kwon JN and Lee WB (2012): Utility of digital pulse oximetry in the screening of lower extremity arterial disease. Journal of the Korean Surgical Society, 82: 94-100

6. Sethi V, Philips S, Hill MF (2013): Lines and Circles: Pictorial Review of Cross-Sectional Imaging of Active Bleeding and Pseudoaneurysm in the Abdomen and Pelvis. Canadian Association of Radiologists Journal, 64: 36-45.
7. Molina JA, Santamaria VB, Guerrero $\mathrm{R}$ et al. (2013): Spectrum of CT Angiography Findings of Pseudoaneurysm. European Congress of Radiology www.myESR.org

8. Rammell J, Kansal $N$, Bhattacharya $V$ (2017): Management options in the treatment of femoral pseudoaneurysms secondary to intravenous drug abuse: A case series. International Journal of Surgery Case Reports, 36: 30-33.

9. Irie Y, Kondo S, Rokkaku K et al. (2017): Obturator foramen bypass for infective groin caused by closure device. Asian Cardiovascular and Thoracic Annals, 25(2): 131-133.

10. Jayaraman $S$, Richardson $D$, Conrad $M$ et al. (2012): Mycotic Pseudoaneurysms Due to Injection Drug Use: A Ten-Year Experience. Annals of Vascular Surgery, 26: 819-824. 